\title{
La excursión a la naturaleza o práctica de campo: una necesidad en la preparación metodológica de los docentes para contribuir al desarrollo local ${ }^{1}$
}

\author{
Lic. Yamilet López Felipe ${ }^{2}$ \\ Dr. Mikel Moreno Hernández $z^{3}$ \\ Dra. Nancy Andreu Gómez ${ }^{4}$
}

\section{RESUMEN}

La incorporación de la excursión geográfica como una de las principales formas de organización docente para el estudio de la localidad y, dentro de sus variantes, la excursión a la naturaleza o práctica de campo, constituye un reto para los diferentes sistemas educativos y una vía para contribuir con la gestión del conocimiento al desarrollo local. En la investigación que se presenta se persigue, a través de las preparaciones metodológicas, contribuir a la preparación de docentes para lograr tal propósito. Se emplearon métodos del nivel teórico y empírico, que permitieron arribar a conclusiones de la conveniencia de usar esta vía para lograr su preparación.

Palabras clave: Excursión geográfica, práctica de campo, preparación metodológica, desarrollo local.

The trip to the nature or practice of field: a necessity in the methodological preparation of the educational ones to contribute to the local development

\section{ABSTRACT}

The incorporation of the geographical trip as one in the main ways of educational organization for the study of the town and inside their variants: the trip to the nature or field practice constitutes a challenge for the different educational systems and a road to contribute with the administration from the knowledge to the local development. In the investigation

I El artículo forma parte de los proyectos "Sistema de acciones educativas medio ambientales del Centro Universitario Municipal (CUM) Placetas, en su entorno comunitario", y "Apoyo al desarrollo local comunitario del municipio de Placetas desde la Universidad Central "Marta Abreu' de Las Villas".

2 Licenciada en Educación. Profesora Asistente, Universidad Central "Marta Abreu" de Las Villas (Centro Universitario Municipal. Placetas),Cuba.E-mail: yalfelipe@uclv.cu

3 Doctor en Ciencias Pedagógicas. Profesor Asistente, Universidad Central "Marta Abreu" de las Villas, Cuba. E-mail: mikelm@uclv.cu

4 Doctora en Ciencias Pedagógicas. Profesora Titular, Universidad Central "Marta Abreu" de las Villas, Cuba.E-mail: nancya@uclv.cu 
that is presented it is pursued through the methodological preparations to contribute to the preparation of educational to achieve such a purpose. Methods of the theoretical and empiric level were used that allowed to arrive to conclusions of the convenience of using this road to achieve their preparation.

Keywords: Geographical trip, field practice, methodological preparation, local development.

\section{La preparación metodológica, una vía de superación de los docentes}

El perfeccionamiento del Sistema Nacional de Educación ha sido un proceso permanente a lo largo de los años, que ha impuesto la necesidad de ir proyectando soluciones a los problemas encontrados. Investigaciones realizadas por el Instituto Central de Ciencias Pedagógicas (ICCP) en el país, entre 2002 y 2013, sobre el contenido de la educación y los planes de estudios, han demostrado que: "Existe una sobrecarga de contenidos en las diferentes asignaturas y de actividades en los horarios, en detrimento de los espacios para la sistematización de aquellos objetivos que son primordiales para los alumnos en cada nivel educativo, además, se redujeron notablemente las frecuencias para la realización de actividades tan importantes $(. .$.$) y$ los espacios para el intercambio entre los alumnos" (García, 2013, p.I).

Según estas investigaciones de estudios teóricos y empíricos del currículo en las distintas educaciones, se debe reflexionar, entre otras, en el número de frecuencias semanales promedio muy elevada para dejar tiempo de autopreparación e investigación a estudiantes y docentes; la obligatoriedad de las actividades complementarias; la ausencia en estas y en otros programas de suficiente correspondencia con el contexto, las necesidades y características de cada territorio y escuela (García, 2013), en circunstancias que, por el contrario, la escuela tiene como finalidad prestar servicios educativos de calidad, adaptados a las necesidades y prioridades del desarrollo de capacidades de las personas como agentes de su propio destino y del desarrollo de sus localidades.

Para lograr lo anterior, se demanda una transformación en la institución escolar, en la que se favorezca que todo el sistema de actividades y relaciones permitan disponer de espacio para adecuarlo a 
las características de cada territorio, dirigiéndose de forma prioritaria, según García (2013), a:

Desarrollar un proceso de enseñanza aprendizaje tomando en cuenta las vivencias y experiencias de los alumnos, base del aprendizaje de nuevos conocimientos, habilidades y hábitos, en función del desarrollo de capacidades, así como de la formación de actitudes, valores, sentimientos, convicciones y otras experiencias de desarrollo y de aprendizaje, desde una visión interdisciplinaria, adecuados a los niveles de desarrollo real y potencial de los alumnos.

La elevación de la preparación para la vida y del desarrollo de los estudiantes, centrada en los valores humanistas, solidarios y patrióticos que caracterizan la sociedad cubana contemporánea.

Fortalecer la actividad laboral, la cultura y actuación ambientalista a partir del enfoque de desarrollo sostenible [que], entre otros, integran los objetivos de la formación ciudadana de los alumnos.

Perfeccionar las acciones comunitarias y relacionadas con el trabajo educativo preventivo y la atención a la población escolar. (p. 4)

Las Ciencias Naturales, y en particular la Geografía, tienen entre sus objetivos estas prioridades, que se pueden lograr mediante las diferentes formas organizativas del proceso enseñanza-aprendizaje.

En este sentido, se requiere de maestros con elevada calificación y de recursos didácticos suficientes, elementos que, por diversas razones, se han ido deteriorando y que constituyen un reto para los diferentes sistemas educativos. La excursión geográfica como una de las principales formas de organización docente $y$, dentro de sus variantes, la excursión a la naturaleza o práctica de campo, puede ser utilizada en las preparaciones metodológicas como una vía que contribuya a la preparación de los docentes en la gestión del conocimiento en función de la educación para el desarrollo local.

Son muchos los autores que, por la importancia de la temática, han trabajado en el tema de los recursos didácticos de la enseñanza 
de la Geografía, entre ellos: Lamadrid (1992), Sánchez (I996), Pulgarín (1998), Bosque (2004), García (2004), Rojas y Valdés (2015), Barea, Cruz y Carrillo (2017), profundizado en la excursión geográfica o alguna de sus modalidades. Este trabajo pretende aportar una propuesta de preparación de excursiones a la naturaleza o prácticas de campo, que contribuyan en las preparaciones metodológicas a potenciar a los docentes en la planificación de estas actividades, garantizando un mayor alcance pedagógico para gestionar el conocimiento en torno a la localidad y la educación para el desarrollo local.

\section{La excursión geográfica como forma de organización docente}

La excursión geográfica es una importante forma de organización docente para el estudio de la localidad y una vía de gestión del conocimiento para contribuir a la educación para el desarrollo local. Los estudiantes tienen la oportunidad de interactuar directamente con el medio ambiente y convertirse en observadores enérgicos de hechos, objetos, fenómenos y procesos geográficos, convirtiendo la realidad que los rodea en un importante medio de enseñanza. Además, permite vincular $y$ aplicar los conocimientos y habilidades adquiridos a nuevas situaciones que están ocurriendo en las localidades en torno al desarrollo local.

Durante la excursión, los estudiantes se forman representaciones, adquieren el contenido sobre los objetos y fenómenos naturales y económicos que sirven de base para la formación de los conceptos físicos y económico-geográficos, insumos útiles para gestionar su propio conocimiento y usar estos en función de su educación para contribuir al desarrollo local.

La excursión geográfica exige una rigurosa planificación y una correcta organización metodológica, debido a las variadas actividades o situaciones de aprendizaje que en ella se efectúan. Autores como Cuétara (2004) y Rojas \& Valdés (2015) coinciden en que se debe tener en cuenta tres etapas: la preparación, la ejecución y la conclusión o culminación. Para estos autores la etapa preparatoria incluye la 
planificación, en la que el profesor realiza una serie de actividades previas para organizar el trabajo docente con los escolares y crear las condiciones para el inicio de la excursión.

Las actividades previas juegan un papel muy importante para garantizar los resultados de las etapas siguientes; dentro de sus tareas esenciales está la determinación clara de los objetivos, constituyendo la base que orienta el desarrollo y la evaluación de la excursión.

En la etapa de ejecución es imprescindible que el profesor asegure que todos los alumnos participen en las actividades planificadas y que trabajen de forma independiente con la guía de aprendizaje. Los estudiantes deben ir registrando todos los datos y muestras recolectadas durante la excursión para luego procesarlos y confeccionar el resumen o informe.

Las principales actividades para esta etapa de ejecución consisten, según Cuétara (2004), en:

- Orientación del mapa sobre el terreno y orientación del lugar.

- Realización de las tareas planificadas en la guía de aprendizaje.

- Observación de los objetos y fenómenos geográficos.

- Anotación de los datos correspondientes.

- Colección de muestras de ejemplares relacionados con el trabajo que se realiza.

- Comprobación parcial de los resultados obtenidos en cada estación (p. I4I).

De regreso en la escuela se efectuará la etapa de culminación o conclusión.

Para desarrollar una correcta excursión y obtener resultados satisfactorios, es preciso aprovechar las potencialidades y, a la vez, las debilidades que ofrece la localidad que rodea la escuela para estimular en los estudiantes el interés hacia la naturaleza y su protección, y así contribuir en la gestión del conocimiento de un desarrollo local sostenible y una correcta educación para enfrentar los retos que debemos asumir con tal desarrollo. 
La excursión geográfica, como una de las principales formas de organización docente para el estudio de la localidad, según Cuétara (2004) refiere, se puede interpretar en sus tres variantes: la excursión a la naturaleza o práctica de campo, visita dirigida y caminata docente.

Por el interés en esta investigación profundizaremos en la excursión a la naturaleza o práctica de campo.

\section{La excursión a la naturaleza o práctica de campo: Una vía de gestión del conocimiento para contribuir a la educación para el desarrollo local}

La escuela tiene como finalidad prestar servicios educativos de calidad, que estén adaptados a las necesidades y prioridades del desarrollo de las capacidades de las personas, como agentes de su propio destino y del desarrollo de sus localidades. Apuesta por una institucionalidad ágil pero sólida, que le permita gestionar adecuadamente su proyecto educativo.

La práctica de campo propicia contribuir con la educación ambiental de los estudiantes al estimular el amor por la naturaleza y la protección del medio ambiente, al formar el carácter de los estudiantes mediante el trabajo en grupo, con disciplina y respeto a sus coetáneos; permite, al realizar las actividades, satisfacer la curiosidad estimulando el pensamiento creativo, tan necesario para lograr iniciativas de desarrollo local sostenibles.

Esta forma de organización del proceso docente, al desarrollarse fuera de la escuela, posee características diferenciadas a las del aula. El estudiante tiene la posibilidad de vincular los contenidos con la localidad, espacio idóneo para establecer relaciones entre los componentes físico-geográficos, económico-geográficos y sociales, así como para resolver problemas que se presentan en la vida práctica y que constituye un principio inviolable de la enseñanza de la Geografía. Además permite al alumno una participación más activa e independiente en su trabajo, facilita conocer la ubicación y desarrollo de objetos y fenómenos geográficos naturales en el espacio en que 
ocurren, así como otros creados por el hombre, lo que contribuye a lograr en los estudiantes un perfil de capacidades de acuerdo con los objetivos de aprendizaje, caracterizado por manejar una comprensión básica del desarrollo local y de sus relaciones con el entorno aplicada a su propia realidad.

Al salir de la escuela los alumnos desarrollan habilidades, sobre todo las relacionadas con la observación, descripción, explicación, lectura de mapas, confección de planos, trazado de croquis, esquemas y gráficas, lo que les permite analizar los objetos geográficos y reflexionar que los componentes de la naturaleza se presentan interrelacionados, formando unidades territoriales naturales (complejos), los que en su orden están relacionados entre sí, a partir de sus formas genéticas y dinámicas. Este tipo de actividad docente es un sistema didáctico con objetivos específicos y métodos de trabajo, constituyendo la base del trabajo la asimilación de conocimientos mediante la observación directa, combinada con el trabajo independiente de los escolares.

Esta variante requiere de una rigurosa planificación, en la que, según Cuétara (2004), se debe concebir un plan que contemple:

- Tema de la excursión.

- Objetivos generales.

- Lugar y fecha de su realización.

- Hora de salida y de regreso.

- Itinerario o marcha-ruta, con sus estaciones.

- Objetivos específicos de cada estación.

- Actividades a realizar por los estudiantes en cada estación.

- Organización de los alumnos para el desarrollo del trabajo y tareas asignadas a cada uno.

- Evaluación de la actividad (p. I44).

Se presentan a continuación dos ejemplos de planificación de excursiones a la naturaleza o prácticas de campo, que pueden ser utilizadas en las preparaciones metodológicas de los docentes como una vía para contribuir a la gestión del conocimiento en función de la educación para el desarrollo local. 


\section{Ejemplo \#1}

Tema de la excursión: Visita al nacimiento del río Zaza. Situación medioambiental.

Objetivos generales:

- Valorar la situación medio ambiental del nacimiento del río Zaza, así como la necesidad de su cuidado y protección a partir de los beneficios que este proporciona y el papel que juega en la vida del hombre.

- Localizar objetos, fenómenos y procesos geográficos que tienen lugar en el espacio que ocupa el nacimiento del río Zaza.

- Caracterizar el espacio geográfico que ocupa el río Zaza, tomando como referencia su situación geográfica y matemática, su extensión y límites, cuenca hidrográfica, parteaguas, afluentes, cauce, embalses, uso de los recursos hídricos.

- Confeccionar croquis del área estudiada.

Contenidos a trabajar:

- Recursos hídricos, río estacional, curso superior de un río cubano, nacimiento del río, cuenca hidrográfica, parte aguas, afluentes, cauce, embalse, uso de los recursos hídricos.

- Meteorización, erosión, acumulación, formas de relieve local.

- Población, urbanización, falsa urbanización, contaminación ambiental, tipos de contaminación.

- Uso del suelo, actividades agrícola, actividades económicas.

- Vegetación, reforestación, plantas exóticas invasoras.

- Identidad, toponimia de la localidad, barrios y consejos populares, historia local.

Lugar y fecha de su realización: Parque infantil Camilo Cienfuegos, ubicado al oeste, noroeste de la ciudad de Placetas (finalizando la Unidad 5 del programa Geografía de Cuba, noveno grado).

Hora de salida y de llegada: 7:30 am - I:30 pm. 
Itinerario o marcha-ruta, con sus estaciones.

Itinerario: Bosque de eucalipto al nacimiento del río Zaza, de ahí a la elevación al oeste del Aserrío, concluyendo en el límite del barrio La Mina y el Reparto Amador.

Estaciones:

Primera estación: Bosque de eucalipto.

Segunda estación: Nacimiento del río Zaza.

Tercera estación: Elevación al oeste del Aserrío.

Cuarta estación. Límite del barrio La mina y el reparto Amador (E1 Mondongo).

Objetivos específicos de cada estación.

Objetivos específicos primera estación: Bosque de eucalipto.

- Caracterizar el espacio geográfico tomando como referencia su situación geográfica y matemática, su extensión y límites, cuenca hidrográfica, parteaguas, cauce, uso de los recursos hídricos, vegetación, especies exóticas invasoras, impacto de la actividad humana.

- Localizar objetos, fenómenos y procesos geográficos que tienen lugar en el bosque de eucalipto.

- Confeccionar croquis de la estación.

Actividades a realizar por los estudiantes en la primera estación.

Organización de los alumnos para el desarrollo del trabajo y tareas asignadas a cada uno

- Se orienta a los estudiantes a ubicarse según los puntos cardinales y posteriormente hacer uso de la hoja topográfica para determinar coordenadas. Pueden hacer uso de la tecnología móvil para ello.

- Los estudiantes deben observar: Parte agua, inclinación del relieve, río intermitente, impacto de la actividad humana, tipo de vegetación existente, reforestación. 
- Se inicia por el equipo de trabajo la elaboración del croquis. Objetivos específicos segunda estación: Nacimiento del río Zaza.

- Caracterizar el espacio geográfico que ocupa el río Zaza, tomando como referencia la ubicación de las coordenadas del lugar del nacimiento del río, comportamiento de las precipitaciones, tipo de vegetación, reforestación, plantas exóticas invasoras, uso del suelo, tipo de suelo, actividades económicas, impacto de la actividad humana. Características del cauce, calidad del agua.

Actividades a realizar por los estudiantes en la segunda estación.

Organización de los alumnos para el desarrollo del trabajo y tareas asignadas a cada uno.

- Ubicación de las coordenadas del lugar. Se realiza un análisis de las precipitaciones promedio de los últimos tres años y se llega a conclusiones (se utiliza el registro del pluviómetro allí existente).

- Observar: Nacimiento del río. Punto de control de precipitaciones. Tipo de vegetación. Reforestación, plantas exóticas invasoras, uso del suelo, tipo de suelo, actividades económicas, impacto de la actividad humana. Características del cauce, calidad del agua.

- Intercambio con los compañeros que atienden el control de precipitaciones sobre la situación del área. Se realiza la medición del nivel del manantial según la escala y se mide el ancho del cauce.

Objetivos específicos tercera estación: Elevación al oeste del Aserrío.

- Caracterizar el espacio geográfico que ocupa el río Zaza, tomando como referencia: Principales actividades económicas. Urbanización y falsa urbanización, erosión de los suelos, tipo de vegetación, inclinación del relieve. Roca serpentina y zona de sabana. Impacto de la actividad humana.

- Confeccionar croquis de la estación.

Actividades a realizar por los estudiantes:

Organización de los alumnos para el desarrollo del trabajo y tareas asignadas a cada uno. 
- Observar: Principales actividades económicas. Urbanización y falsa urbanización, erosión de los suelos, tipo de vegetación, inclinación del relieve. Roca serpentina y zona de sabana. Impacto de la actividad humana.

- Los estudiantes elaboran un croquis y determinan las zonas con importante actividad económica y con áreas de viviendas. Se describe el tipo de rocas y el perfil del suelo, utilizando el lugar donde este es visible.

Objetivos específicos cuarta estación. Límite del barrio La mina y el reparto Amador (El Mondongo).

- Caracterizar el espacio geográfico que ocupa el río Zaza, teniendo en cuenta el tamaño del cauce, la velocidad de la corriente. Principales efectos negativos producto de la actividad humana. Actividades económicas. Impacto de la actividad humana. Características del cauce, calidad del agua, peligros y riesgos relacionados con el río. Toponimia del lugar, características de los afluentes, erosión, deposición, identidad, historia local.

- Intercambio con pobladores del efecto de las inundaciones y de la historia del lugar.

Actividades a realizar por los estudiantes:

Organización de los alumnos para el desarrollo del trabajo y tareas asignadas a cada uno.

- Observar: Actividades económicas. Impacto de la actividad humana. Características del cauce, calidad del agua, peligros y riesgos relacionados con el río. Toponimia del lugar, características de los afluentes, erosión, deposición, identidad, historia local.

- En esta estación los estudiantes miden el tamaño del cauce la velocidad de la corriente. Definen los principales efectos negativos producto de la actividad humana. Intercambian con pobladores acerca del efecto de las inundaciones y de la historia del lugar.

Organización de los alumnos para el desarrollo del trabajo y tareas asignadas a cada uno. 
Evaluación de la actividad. Los participantes elaboran los resultados definidos para cada grupo. De ser posible, se debe promover conformar presentaciones, catálogos de fotografías, perfiles, muestrario $\mathrm{u}$ otros que ayuden a tener evidencias de las acciones realizadas.

\section{Ejemplo \# 2. Alturas del norte de Placetas.}

Tema de la excursión: Disfrutando las bellezas de las alturas del norte de Placetas

- Objetivos generales:

- Valorar la situación medio ambiental de las alturas del norte de Placetas, así como la necesidad de su cuidado y protección a partir de los beneficios que este proporciona y el papel que juega en la vida del hombre.

- Localizar objetos, fenómenos y procesos geográficos.

- Caracterizar el espacio geográfico que ocupa, tomando como referencia su situación geográfica y matemática, su extensión y límites.

- Confeccionar croquis del área estudiada.

Contenidos a trabajar:

- Tipo de relieve, alturas, llanuras y montañas. Valles.

- Carso. Formas de relieve cársico. Meteorización, erosión, acumulación.

- Rocas ígneas, sedimentarias y metamórficas.

- Tipo de suelo. Uso del suelo, actividades agrícola, actividades económicas.

- Vegetación, reforestación, plantas exóticas invasoras, bosque en galerías.

- Identidad. Toponimia de la localidad, historia local.

Lugar y fecha de su realización: Secundaria I3 de marzo. (Finalizando la Unidad 2 del programa Geografía de Cuba, noveno grado). 
Hora de salida y de llegada: 7:30 am - I:30 pm.

Itinerario o marcha-ruta, con sus estaciones.

Itinerario: Loma de la Vallett, Loma de la Vigía, Loma de los Fósiles.

Estaciones:

Primera estación: Loma de la Vallett.

Segunda estación: Loma de la Vigía.

Tercera estación. Loma de los Fósiles.

Objetivos específicos de cada estación.

Objetivos específicos primera estación: Loma de la Vallett.

- Caracterizar el espacio geográfico que ocupa la Loma de la Vallett, tomando como referencia situación geográfica y matemática, orientación de las elevaciones, curvas de nivel, tipo de rocas, principales puntos de referencias. Disposición general del relieve. Tipo de vegetación. Identidad. Toponimia de la localidad, historia local.

Actividades a realizar por los estudiantes en la primera estación.

Observar: Orientación de las elevaciones, curvas de nivel, tipo de rocas, principales puntos de referencia. Disposición general del relieve. Tipo de vegetación.

Se orienta a los estudiantes a ubicarse según los puntos cardinales $y$, posteriormente, hacer uso de la hoja topográfica para determinar coordenadas. Pueden hacer uso de la tecnología móvil para ello. Se toman muestras de rocas y de vegetación para enriquecer el informe, y se realizan fotografías en función de las características del relieve. Identidad. Es necesario profundizar en la toponimia de la localidad e historia local asociada con la zona.

Objetivos específicos segunda estación: Loma de la Vigía.

- Caracterizar el espacio geográfico que ocupa la Loma de la Vigía, tomando como referencia situación geográfica y matemática, 
orientación de las elevaciones, curvas de nivel, tipo de rocas, tipo de vegetación. Tipo de suelo. Principales puntos de referencias. Disposición general del relieve. Identidad. Toponimia de la localidad, historia local.

Actividades a realizar por los estudiantes en la segunda estación.

Se orienta a los estudiantes a ubicarse según los puntos cardinales y, posteriormente, hacer uso de la hoja topográfica para determinar coordenadas.

Observar: Orientación de las elevaciones, curvas de nivel, tipo de rocas, tipo de vegetación referencias. Tipo de suelo. Disposición general del relieve. Identidad. Toponimia de la localidad, historia local.

Objetivos específicos tercera estación: Loma de los Fósiles.

Caracterizar el espacio geográfico que ocupa la Loma de los Fósiles, tomando como referencia situación geográfica y matemática, orientación de las elevaciones, curvas de nivel, tipo de rocas, tipo de vegetación. Tipo de suelo. Principales puntos de referencias. Disposición general del relieve. Identidad. Toponimia de la localidad, historia local.

Actividades a realizar por los estudiantes en la tercera estación.

Se orienta a los estudiantes a ubicarse según los puntos cardinales y, posteriormente, hacer uso de la hoja topográfica para determinar coordenadas.

Observar: Orientación de las elevaciones, curvas de nivel, tipo de rocas, tipo de vegetación referencias. Tipo de suelo. Disposición general del relieve. Identidad. Toponimia de la localidad, historia local.

En esta estación se debe priorizar la observación de las rocas sedimentarias, la búsqueda de fósiles y su valor para conocer el pasado de la zona.

Organización de los alumnos para el desarrollo del trabajo y tareas asignadas a cada uno.

Evaluación de la actividad. Se debe ejecutar la exposición del resultado obtenido; hay que insistir en la elaboración de muestras, croquis, etc. 


\section{Conclusiones}

La excursión a la naturaleza o práctica de campo constituye una forma importante de organización docente que puede ser aprovechada en la gestión del conocimiento de la localidad, con el fin de contribuir a la educación para el desarrollo local.

La aplicación de esta forma de organización docente demanda preparación, de ahí la necesidad de instrumentarlas en las preparaciones metodológicas en función de las potencialidades locales.

\section{Referencias bibliográficas}

Barea, Y., Cruz, M. y Carrillo, H. (2017). Procedimientos metodológicos para la realización de excursiones docentes integradoras en Ciencias Naturales. Educación y Sociedad, 25(3), I08-II7.

Bosque, R. (2004). Propuesta inicial de estructuración didáctica de la excursión docente en la enseñanza de las Ciencias Naturales. Tesis en opción al grado científico de Doctor en Ciencias Pedagógicas. Instituto Superior Pedagógico "Enrique José Varona”, La Habana.

Cuétara, R. (2004). Hacia una Didáctica de la Geografía Local. La Habana: Editorial Pueblo y Educación.

García, A. (2004). El itinerario geográfico como recurso didáctico para la valoración del paisaje. Didáctica geográfica, (6), 79-95. Recuperado de https://repositorio.uam.es/bitstream/handle/I0486/6666I6/ itinerario garcia_DG_2004.pdf? sequence $=\mathrm{I}$ \&isAllowed $=\mathrm{y}$

García, L. J. (2013). Proceso de perfeccionamiento del sistema educacional desde su concepción teórico-metodológica. Estado actual y perspectivas (Documento Base). La Habana: ICCP.

Lamadrid, J. (1992). Metodología para la realización de las prácticas de campo físico geográficas. Tesis en opción al grado científico de Doctor en Ciencias Pedagógicas. Instituto Superior Pedagógico "Enrique José Varona”, La Habana.

Pulgarín, R. (1998). La excursión escolar como estrategia didáctica en la enseñanza de la geografía. La Gaceta Didáctica, l(2), I-7. Recuperado de http://aulasvirtuales2.uruguayeduca.edu.uy/pluginfile.php/309II/ mod forum/attachment/24056/P000I File La\%20salida\%20 de $\% 20$ campo $\% 20$ como $\% 20$ estrategia $\% 20$ did $\%$ C3\%AIctica.pdf 
Rojas, E. y Valdés, M. (2015). La excursión geográfica, una propuesta para su desarrollo desde la asignatura Geografía en décimo grado del Instituto Vocacional de Ciencias Exactas "Federico Engels". Revista Mendive, 13 (2), I57-I65.

Sánchez, J. (1996). Recursos didácticos para una enseñanza renovada de la Geografía. Didáctica Geográfica, (I), 7-I4. Recuperado de http://www. age-geografia.es/didacticageografica/index.php/didacticageografica/ article/download/I23/I27 